\title{
Second-order gravitational self-force
}

\author{
Adam Pound ${ }^{1}$ \\ ${ }^{1}$ School of Mathematics, University of Southampton, Southampton, United Kingdom, SO17 1 BJ
}

(Dated: October 16, 2018)

\begin{abstract}
Using a rigorous method of matched asymptotic expansions, I derive the equation of motion of a small, compact body in an external vacuum spacetime through second order in the body's mass (neglecting effects of internal structure). The motion is found to be geodesic in a certain locally defined regular geometry satisfying Einstein's equation at second order. I outline a method of numerically obtaining both the metric of that regular geometry and the complete second-order metric perturbation produced by the body.
\end{abstract}

PACS numbers: 04.20.-q, 04.25.-g, 04.25.Nx, 04.30.Db

Introduction. The governing equation of general relativity, the Einstein field equation (EFE), describes how bodies influence spacetime curvature and move within the resultant curved geometry. Yet, since the seminal work of Einstein, Infeld, and Hoffman in 1938 [1], study of this nonlinear problem of motion has largely focused on the post-Newtonian limit of slow motion and weak fields. In the strong-field regime, bodies have instead typically been approximated as test bodies moving in a spacetime that is unaffected by them. Only within the last fifteen years 2, 3] has there arisen an analytical description of the gravitational backreaction: the body's perturbative effect on spacetime geometry and that perturbation's effect on the body's motion. In the case of a small mass $m$, this backreaction is called the gravitational self-force, and it is now well understood at linear order in $m$ [4 7]. Beyond its foundational role, the self-force is also potentially of great astrophysical importance, as it describes the evolution of extreme-mass-ratio inspirals (EMRIs), in which a stellar black hole or neutron star spirals into a supermassive black hole. Such systems are predicted to be key sources for the planned gravitational wave detector LISA [8], and they will afford both a unique probe of strong-field dynamics and a map of the spacetime near black holes. The self-force also provides an essential point of comparison with other treatments of the problem of motion: it complements post-Newtonian theory [9, 10] and fully nonlinear numerical simulations [10, 11], both of which are ill-suited to extreme mass ratios; and it fixes mass-dependent parameters in Effective One Body (EOB) theory [12 14].

However, to extract orbital parameters from a waveform emitted by an EMRI, one requires a theoretical description accurate to second order in the small mass, as shown by either naive or rigorous [15] scaling arguments. Furthermore, comparisons with numerical simulations suggest that the second-order self-force would provide a highly accurate description of intermediate-massratio binaries and even a reasonably accurate description of similar-mass binaries [10, 11], both of which should soon be observed by the ground-based detector Advanced LIGO [16, 17]. The second-order force would also fix
EOB parameters quadratic in $m$. Although some work on the second-order problem has been done [18, 19], it was performed in an impractical gauge, with no clear means of calculating the force or the perturbation producing it, and the basis of its approach was problematic [57, 20]. Detweiler has recently [21] examined the general features of the second-order problem, but he made use of ill-defined equations and assumed (rather than derived) the equation of motion and a regularized second-order stress-energy tensor. Harte 22] has derived an equation of motion valid at all orders, but his derivation does not apply to the motion of black holes. None of these studies has provided a definite expression for, or means of calculating, the physical second-order metric perturbation or the piece of it that determines the motion.

In this letter, I present the first complete description, explicitly determining both the equation of motion and the metric perturbation. I use the self-consistent formalism presented in [6]. This formalism incorporates the small body's finite size, involving no infinities or regularization; it defines a worldline $\gamma$ that reflects the body's motion (even a black hole's) on any timescale; it determines the equation of motion directly from the EFE, with no further axioms; and it presents the perturbative EFEs in a hyperbolic form convenient for numerical implementation. For simplicity, I take the body to be spherical and nonspinning, neglecting higher multipole moments. I work in units of $G=c=1$. Greek indices range from 0 to 3. Lowercase Latin indices refer to spatial coordinates. Further details will appear in a follow-up article [20].

Self-consistent formalism. I combine two approximate solutions to the EFE, utilizing the method of matched asymptotic expansions [7, 23, 25]. Suppose $\mathrm{g}_{\mu \nu}(\epsilon)$ is an exact solution containing the small body on a manifold $\mathcal{M}$, where $\epsilon$ is an expansion parameter that counts powers of the body's mass. Now let $r$ be some measure of distance from the body and $\mathcal{R}$ represent the spacetime's lengthscales, excluding those of the body itself. For $r \sim$ $\mathcal{R}$, well outside the body, in any global coordinates $x^{\alpha}$ in a vacuum region $\Omega$ (e.g., Boyer-Lindquist coordinates of the supermassive black hole in an EMRI), I use the outer expansion $\mathrm{g}_{\mu \nu}\left(x^{\alpha}, \epsilon\right)=g_{\mu \nu}\left(x^{\alpha}\right)+h_{\mu \nu}\left(x^{\alpha}, \epsilon\right)$ on a man- 
ifold $\mathcal{M}_{E} \cdot\left(g_{\mu \nu}, \mathcal{M}_{E}\right)$ defines an external background spacetime with no small body in it, and $h_{\mu \nu}(x, \epsilon)=$ $\sum_{n>1} \epsilon^{n} h_{\alpha \beta}^{(n)}(x ; \gamma(\epsilon))$ describes perturbations due to the body, whose motion in $\mathcal{M}_{E}$ is represented by $\gamma$. For $r \sim \epsilon \mathcal{R}$, very near the body, the metric varies rapidly, and there, in any coordinates $\left(T, X^{a}\right)$ approximately centered on the body, I use the inner expansion $\mathrm{g}_{\mu \nu}\left(T, X^{a} / \epsilon, \epsilon\right)=$ $g_{I \mu \nu}\left(T, X^{a} / \epsilon\right)+\sum_{n \geq 1} \epsilon^{n} H_{\mu \nu}^{(n)}\left(T, X^{a} / \epsilon\right)$ on a manifold $\mathcal{M}_{I} .\left(g_{I \mu \nu}, \mathcal{M}_{I}\right)$ is the body's spacetime were it isolated, and $H_{\mu \nu}^{(n)}$ describes perturbations due to interactions with the external spacetime. The scaled coordinates $X^{a} / \epsilon$ serve to keep the body's mass and size fixed in the limit $\epsilon \rightarrow 0$, sending all other distances toward infinity; the use of a single rescaling factor makes the approximation most appropriate for compact bodies, in which the linear dimension is comparable to the mass (in geometrized units). Scaling only distances, not $T$, is equivalent to assuming the body possesses no fast internal dynamics.

In a buffer region around the body, defined by $\epsilon \ll$ $r / \mathcal{R} \ll 1$, either expansion may be used, and since they approximate the same metric, they must agree: the inner expansion can be expressed in unscaled coordinates and then expanded for $r / \mathcal{R} \gg \epsilon$, the outer expansion can be expanded for $r / \mathcal{R} \ll 1$, and the two results must match order by order in $r$ and $\epsilon$. It follows [6] that $h_{\mu \nu}^{(n)} \sim$ $1 / r^{n}+O\left(r^{-n+1}\right)$, and the $1 / r^{n}$ term is determined by the $(n-1)$ th multipole moment of $g_{I \mu \nu}$. If $r$ is a radial coordinate centered on $\gamma$ and this form of $h_{\mu \nu}^{(n)}$ holds true, then both the body - in the full spacetime - and $\gamma$-in the background spacetime - lie in the region surrounded by the buffer. If all mass dipole terms also vanish in this coordinate system, then the body is appropriately centered on $\gamma$.

In standard perturbation theory, the linearized EFE would constrain $\gamma$ to be a geodesic in $g_{\mu \nu}$. To avoid this, I write the full, nonlinear EFE in $\Omega$ in relaxed form by imposing the Lorenz gauge condition on the whole of $h_{\mu \nu}$, rather than on each term $h_{\mu \nu}^{(n)}$ :

$$
g^{\nu \rho} \nabla_{\rho} \bar{h}_{\mu \nu}=0, \quad \text { where } \bar{h}_{\mu \nu} \equiv h_{\mu \nu}-\frac{1}{2} g_{\mu \nu} g^{\rho \sigma} h_{\rho \sigma} .
$$

The exact vacuum EFE in $\Omega, \mathrm{R}_{\mu \nu}=0$, then splits into a sequence of wave equations,

$$
\begin{aligned}
& E_{\mu \nu}\left[h^{(1)}\right]=0, \\
& E_{\mu \nu}\left[h^{(2)}\right]=2 \delta^{2} R_{\mu \nu}\left[h^{(1)}\right],
\end{aligned}
$$

etc., where $E_{\mu \nu}$ is the linear wave-operator $E_{\mu \nu}[h]=$ $\square h_{\mu \nu}+2 R_{\mu}{ }^{\rho}{ }_{\nu}{ }^{\sigma} h_{\sigma \rho}, R_{\mu \nu \rho \sigma}$ is the Riemann tensor of the external background $g_{\mu \nu}$, and $\delta^{2} R_{\mu \nu}$ is the part of the Ricci tensor quadratic in the metric perturbation. No stress-energy tensor for the body appears here, since the body lies outside $\Omega$. Equations (2) and (3) can be solved for arbitrary $\gamma$, the equation of motion of which is then determined by the gauge condition. That condition will involve $\gamma^{\prime}$ 's $\epsilon$-dependent acceleration $a^{\mu}(\tau, \epsilon)$, where $\tau$ is proper time on $\gamma$, and I split Eq. (1) into a sequence of equations for each $h_{\mu \nu}^{(n)}$ by substituting into it an expansion $a^{\mu}=\sum_{n \geq 0} \epsilon^{n} a^{(n) \mu}$.

Outer expansion. In the buffer region, I construct the most general possible solution to the wave equations (2) and (3) and gauge condition (11). That local solution is then used to construct a global solution. I work in Fermi-Walker coordinates $\left(t, x^{a}\right)$ centered on $\gamma$, where $t$ coincides with $\tau$ on $\gamma$ and $x^{i}$ are Cartesian coordinates on the spatial submanifold transverse to $\gamma$ at time $t$. I assume each $h_{\mu \nu}^{(n)}$ can be expanded for small geodesic distance $r \equiv \sqrt{\delta_{i j} x^{i} x^{j}}$. So at first order,

$$
h_{\mu \nu}^{(1)}\left(t, x^{a}\right)=\sum_{m \geq-1, \ell \geq 0} r^{m} h_{\mu \nu L}^{(1, m, \ell)}(t) \hat{n}^{L},
$$

where $L \equiv i_{1} \cdots i_{\ell}, n^{i} \equiv x^{i} / r$ is a radial unit vector, and $\hat{n}^{L} \equiv n^{\left\langle i_{1}\right.} \cdots n^{\left.i_{\ell}\right\rangle}$ is the symmetric and trace-free (STF) combination of $\ell$ such unit vectors; this decomposition in terms of $\hat{n}^{L}$ is equivalent to an expansion in spherical harmonics [26]. Substituting (4) into (2), one finds that each term in Eq. (4) must satisfy a Poisson equation, $\sum_{\ell} \partial^{i} \partial_{i}\left(r^{m} \hat{n}^{L}\right) h_{\mu \nu L}^{(1, m, \ell)}(t)=r^{m-2} \sum_{\ell} S_{\mu \nu L}^{(1, m, \ell)}(t) \hat{n}^{L}$. The source $S_{\mu \nu L}^{(1, m, \ell)}$ is a linear combination of the lower-order terms $h_{\mu \nu L^{\prime}}^{\left(1, m^{\prime}<m, \ell^{\prime}\right)}$; it involves derivatives, $a^{\mu}$, and $R_{\mu \nu \rho \sigma}$. The general solution to this Poisson equation consists of a homogeneous solution, comprising the single mode $h_{\mu \nu L_{H}}^{\left(1, m, \ell_{H}\right)}$ where $\ell_{H}=m$ for $m \geq 0$ and $\ell_{H}=-m-1$ for $m<0$, plus a particular inhomogeneous solution with modes $h_{\mu \nu L}^{(1, m, \ell) \mathrm{P}}$ directly proportional to $S_{\mu \nu L}^{(1, m, \ell)}$. Given how $S_{\mu \nu L}^{(1, m, \ell)}$ is constructed, clearly each $h_{\mu \nu L}^{(1, m, \ell) \mathrm{P}}$ is a linear combination of the lower-order modes $h_{\mu \nu L_{H}^{\prime}}^{\left(1, m^{\prime} \leq m, \ell_{H}^{\prime}\right)}$; therefore, the general solution [of the form (4)] to Eq. (2) is wholly determined by the functions $h_{\mu \nu L_{H}}^{\left(1, m, \ell_{H}\right)}(t)$. The first of these, $h_{\mu \nu}^{(1,-1,0)}$, is fixed to be $2 m \delta_{\mu \nu}$, where $m$ is the ADM mass of the inner background $g_{I \mu \nu}[5,[6]$. All the others, $h_{\mu \nu L_{H}}^{\left(1, m \geq 0, \ell_{H}\right)}$, are undetermined at this stage. It will thus prove convenient to split the general solution into two pieces: $h_{\mu \nu}^{(1)}=h_{\mu \nu}^{(1) \mathrm{S}}+h_{\mu \nu}^{(1) \mathrm{R}}$. I define the regular field $h_{\mu \nu}^{(1) \mathrm{R}}$ to comprise all terms involving the undetermined functions $h_{\mu \nu L_{H}}^{\left(1, m \geq 0, \ell_{H}\right)}$. It reads

$$
h_{\mu \nu}^{(1) \mathrm{R}}=h_{\mu \nu}^{(1,0,0)}(t)+r h_{\mu \nu i}^{(1,1,1)}(t) n^{i}+O\left(r^{2}\right) .
$$

The singular field $h_{\mu \nu}^{(1) \mathrm{S}}$ then comprises all the other terms; it is the particular solution obtained by setting $h_{\mu \nu L_{H}}^{\left(1, m \geq 0, \ell_{H}\right)}=0$. It reads

$$
\begin{aligned}
h_{\mu \nu}^{(1) \mathrm{S}}= & \frac{2 m}{r} \delta_{\mu \nu}+h_{\mu \nu i}^{(1,0,1)}(t) n^{i} \\
& +r \sum_{\ell=0,2} h_{\mu \nu L}^{(1,1, \ell)}(t) \hat{n}^{L}+O\left(r^{2}\right),
\end{aligned}
$$


where the functions $h_{\mu \nu L}^{(n, m, \ell)}(t)$ are linear in $m$. I stress that this split is merely a convenient grouping of terms in the general solution in the buffer region, with no impact on the results. When so defined, $h_{\mu \nu}^{(1) \mathrm{S}}$ and $h_{\mu \nu}^{(1) \mathrm{R}}$ are each solutions to the first-order field equations in $\Omega$. $h_{\mu \nu}^{(1) \mathrm{S}}$ can be interpreted as the body's bound field; it is determined solely by the fact that a compact body lies in the region surrounded by the buffer. $h_{\mu \nu}^{(1) \mathrm{R}}$ is a homogeneous (local) solution to the wave equation even at $r=0$, propagating independently of the body; as a homogeneous solution, it can be determined only by global boundary conditions. Imposing the gauge condition determines the isotropic, $\delta_{\mu \nu}$ form of the $m / r$ term given above, as well as determining that the body behaves approximately as a test particle, with constant mass (i.e., $\partial_{t} m=0$ ) and approximately geodesic motion (i.e., $a^{(0) \mu}=0$ ).

One can prove with distributional [5] or Green's function [6] methods that $h_{\mu \nu}^{(1)}$, because of its $m / r$ term, is sourced by the stress-energy tensor of a point particle, $T^{(1) \mu \nu}=\int_{\gamma} m u^{\mu} u^{\nu} \frac{\delta^{4}\left(x^{\alpha}-z^{\alpha}(\tau)\right)}{\sqrt{-g}} d \tau$, where $z^{\mu}(\tau)$ is the parametrization of $\gamma, u^{\mu} \equiv \frac{d z^{\mu}}{d \tau}$, and $g$ is the determinant of $g_{\mu \nu}$. Therefore, at distances in the buffer region or greater, the body appears as a point mass. With this determined, $h_{\mu \nu}^{(1)}$ can be found globally by solving the wave equation $E_{\mu \nu}\left[h^{(1)}\right]=-16 \pi \bar{T}_{\mu \nu}^{(1)}$, where an overbar indicates trace-reversal. Using retarded boundary conditions, for example, the global solution will then fix the locally undetermined field $h_{\mu \nu}^{(1) \mathrm{R}}$. Numerous methods have been used to accomplish this [4]. In the case that $h_{\mu \nu}^{(1)}$ contains no contribution from incoming waves at infinity, doing so determines [6] that at least through order $r$, $h_{\mu \nu}^{(1) \mathrm{R}}$ is the Detweiler-Whiting regular field [27].

The second-order solution proceeds almost identically. I assume an expansion

$$
\begin{aligned}
h_{\mu \nu}^{(2)}\left(t, x^{a}\right)= & \sum_{m \geq-2, \ell \geq 0} r^{m} h_{\mu \nu L}^{(2, m, \ell)}(t) \hat{n}^{L} \\
& +\ln r \sum_{m \geq 0, \ell \geq 0} r^{m} h_{\mu \nu L}^{(2, m, \ln , \ell)}(t) \hat{n}^{L},
\end{aligned}
$$

where the logarithmic terms arise from the correction $\sim m \ln (r / 2 m-1)$ to the light cones in the buffer region (where $r \gg m$ ) [6]. Substituting this into Eq. (3), together with an expansion of $h_{\mu \nu}^{(1)}$ up to order $r^{2}$, and finding the general solution at each order again allows the convenient split $h_{\mu \nu}^{(2)}=h_{\mu \nu}^{(2) \mathrm{R}}+h_{\mu \nu}^{(2) \mathrm{S}}$. However, here I define $h_{\mu \nu}^{(2) \mathrm{R}}$ to comprise not just all terms involving the undetermined functions $h_{\mu \nu L_{H}}^{\left(2, m \geq 0, \ell_{H}\right)}(t)$, but also all terms quadratic in $h_{\mu \nu}^{(1) \mathrm{R}}$. This guarantees that $h_{\mu \nu}^{(2) \mathrm{R}}$ satisfies $E_{\mu \nu}\left[h^{(2) \mathrm{R}}\right]=2 \delta^{2} R_{\mu \nu}\left[h^{(1) \mathrm{R}}\right]$, such that $g_{\mu \nu}+\epsilon h_{\mu \nu}^{(1) \mathrm{R}}+\epsilon^{2} h_{\mu \nu}^{(2) \mathrm{R}}$ satisfies the vacuum EFE through order $\epsilon^{2}$. Explicitly,

$$
h_{\mu \nu}^{(2) \mathrm{R}}=h_{\mu \nu}^{(2,0,0)}(t)+r h_{\mu \nu i}^{(2,1,1)}(t) n^{i}+O\left(r^{2}\right) ;
$$

TABLE I. Components of $\delta m_{\mu \nu}(t)$ in terms of the first-order regular field $h_{\mu \nu}^{(1) \mathrm{R}}(t, r=0)=h_{\mu \nu}^{(1,0,0)}(t)$.

\begin{tabular}{l}
$\delta m_{t t}=-m h_{t t}^{(1,0,0)}-\frac{1}{6} m \delta^{i j} h_{i j}^{(1,0,0)}$ \\
$\delta m_{t a}=-\frac{2}{3} m h_{t a}^{(1,0,0)}$ \\
$\delta m_{a b}=\delta_{a b}\left(\frac{1}{3} m h_{t t}^{(1,0,0)}+\frac{5}{18} m \delta^{i j} h_{i j}^{(1,0,0)}\right)+\frac{1}{3} m h_{\langle a b\rangle}^{(1,0,0)}$ \\
\hline
\end{tabular}

terms quadratic in $h_{\mu \nu}^{(1) \mathrm{R}}$ would appear at order $r^{2}$. The singular field $h_{\mu \nu}^{(2) \mathrm{S}}$, comprising all other terms in the general solution, then reads

$$
\begin{aligned}
h_{\mu \nu}^{(2) \mathrm{S}}= & \frac{1}{r^{2}} \sum_{\ell=0,2} h_{\mu \nu L}^{(2,-2, \ell)} \hat{n}^{L}+\frac{1}{r} \sum_{\ell=1}^{3} h_{\mu \nu L}^{(2,-1, \ell)} \hat{n}^{L}+\frac{2 \delta m_{\mu \nu}}{r} \\
& +\sum_{\ell=1}^{4} h_{\mu \nu L}^{(2,0, \ell)} \hat{n}^{L}+r \sum_{\ell=0,2,3,4,5} h_{\mu \nu L}^{(2,0, \ell)} \hat{n}^{L} \\
& +\ln r\left[h_{\mu \nu}^{(2,0, \ln , 0)}+r h_{\mu \nu i}^{(2,1, \ln , 1)} n^{i}\right]+O\left(r^{2}\right),
\end{aligned}
$$

where $\delta m_{\mu \nu}$ is a mass-like tensor defined on $\gamma$. The explicit terms in this expansion can be found in Ref. [6] through order $r^{0}$ when $a^{\mu}=0$, and they will be written out in full in the follow-up article [20]; the functions $h_{\mu \nu L}^{(2, m, \ell)}(t)$ are linear combinations of $m^{2}, m h_{\mu \nu}^{(1) \mathrm{R}}$, and $\delta m_{\mu \nu}$. The body's dipoles (i.e., those of $g_{I \mu \nu}$ ) would contribute to $h_{\mu \nu}^{(2) \mathrm{S}}$, but I set the spin to zero for simplicity and the mass dipole to zero to ensure that $\gamma$ accurately represents the body's motion. Note that my definition of $h_{\mu \nu}^{(2) \mathrm{R}}$ means that $h_{\mu \nu}^{(2) \mathrm{S}}$ satisfies not Eq. (3), but $E_{\mu \nu}\left[h^{(2) \mathrm{S}}\right]=2 \delta^{2} R_{\mu \nu}\left[h^{(1)}\right]-2 \delta^{2} R_{\mu \nu}\left[h^{(1) \mathrm{R}}\right]$.

As at first order, the gauge condition determines the form of $\delta m_{\mu \nu}$, as given in Table [. along with the firstorder acceleration,

$$
a_{i}^{(1)}(t)=\left.\frac{1}{2} h_{t t i}^{(1,1,1)}(t)\right|_{a^{\mu}=0}-\left.h_{t i, t}^{(1,0,0)}(t)\right|_{a^{\mu}=0} .
$$

The evaluation at $a^{\mu}=0$ is to be performed only at time $t$, leaving the past history of $\gamma$ unchanged; this follows from the presumed expansion of $a^{\mu}$, and it prevents a need for order-reduction [4, [6]. One can show Eq. (10) is equivalent to the geodesic equation in $g_{\mu \nu}+\epsilon h_{\mu \nu}^{(1) \mathrm{R}}$ at order $\epsilon[4]$.

Using the same Green's-function method [6] as at first order, one can straightforwardly prove that the terms involving $\delta m_{\mu \nu}$ have a point source, the trace-reversal of which is given by the effective stress-energy tensor

$$
T_{\mu \nu}^{(2)}=\int_{\gamma} \frac{1}{2} \overline{\delta m}_{\mu \nu}(\tau) \frac{\delta^{4}\left(x^{\alpha}-z^{\alpha}(\tau)\right)}{\sqrt{-g}} d \tau .
$$

The global solution to the second-order EFE sourced by the body can then be obtained numerically via a puncture scheme 28]: Outside a tube $\Gamma$ around the body, one can solve Eq. (3) directly; inside the tube, one can use an 
approximation $\tilde{h}_{\mu \nu}^{(2) \mathrm{S}}$ to $h_{\mu \nu}^{(2) \mathrm{S}}$, given by Eq. (9) without the " $O\left(r^{2}\right)$ " term, and a regular field $\tilde{h}_{\mu \nu}^{(2) \mathrm{R}} \equiv h_{\mu \nu}^{(2)}-\tilde{h}_{\mu \nu}^{(2) \mathrm{S}}$, which satisfies

$$
E_{\mu \nu}\left[\tilde{h}^{(2) \mathrm{R}}\right]=-16 \pi \bar{T}_{\mu \nu}^{(2)}+2 \delta^{2} R_{\mu \nu}\left[h^{(1)}\right]-E_{\mu \nu}\left[\tilde{h}^{(2) \mathrm{S}}\right] .
$$

All divergent terms on the right-hand side cancel, leaving $\tilde{h}_{\mu \nu}^{(2) \mathrm{R}}$ to solve a wave equation with a regular source. At $\Gamma$, the analytical expression for $\tilde{h}_{\mu \nu}^{(2) \mathrm{S}}$ can be added to the numerical solution to Eq. (12) to change variables to the full field. Since $\tilde{h}_{\mu \nu}^{(2) \mathrm{R}}$ will agree with $h_{\mu \nu}^{(2) \mathrm{R}}$ through order $r$, this procedure will also determine $h_{\mu \nu}^{(2,0,0)}(t)$ and $h_{\mu \nu i}^{(2,1,1)}(t)$, which, as we shall find. This puncture scheme can be implemented immediately after transforming Eq. (9) to a desired coordinate system.

Matching to an inner expansion. One could proceed with the same method to find $h_{\mu \nu}^{(3)}$ together with $a^{(2) \mu}$. I instead take a more efficient route by determining $a^{(2) \mu}$ from additional information about the inner expansion. I take the small body to be a Schwarzschild black hole. Since the inner expansion affects the outer one solely through the body's multipole moments, this amounts to neglecting non-monopole moments, as was done in the preceding section. Beyond effects of those moments, the equation of motion I derive will hold for any compact body. I also specify the perturbations to be produced by tidal fields. These fields are of quadrupole order and higher, and while they produce mass and spin perturbations via tidal heating and torquing, dimensional analysis shows those perturbations do not contribute at the orders in $\epsilon$ of interest. With these specifications, I follow the procedure in Ref. [7], insisting that in a suitable mass-centered coordinate system, this inner expansion must equal the outer expansion in Fermi-Walker coordinates when expanded in the buffer region, up to a unique gauge transformation that excludes spatial translations at $\gamma$; this ensures the desired relationship between $\gamma$ and the mass-centered inner expansion. Here 'mass-centered coordinate system' means one in which the mass dipole of $g_{I \mu \nu}$ vanishes along with all even-parity dipole perturbations that behave as a mass dipole, scaling as $1 / r^{2}$ in the buffer region. Other even-parity dipole perturbations are also set to zero in order to leave no residual gauge freedom. Because the Fermi-Walker coordinates are $\epsilon$-dependent, each term in the outer expansion depends on $a^{\mu}$. Hence, prior to matching the metrics, I substitute $a^{\mu}=\sum_{n \geq 0} \epsilon^{n} a_{(n)}^{\mu}$ into $g_{\mu \nu}+\sum_{n \geq 1} \epsilon^{n} h_{\mu \nu}^{(n)}$.

A tidally perturbed Schwarzschild metric is given in Ref. 29] in advanced Eddington-Finkelstein coordinates and a light-cone gauge. The tidal fields are represented by STF tensorial functions of time: $\mathcal{E}_{i j}$ and $\mathcal{B}_{i j}$ for electric- and magnetic-type quadrupole tides and $\mathcal{E}_{i j k}$ and $\mathcal{B}_{i j k}$ for analogous octupole tides; for the powers of $r$ of interest, hexadecapole and higher tidal fiels would appear only at order $\epsilon^{3}$ in the outer expansion. Trans- forming to a suitable Fermi-like coordinate system and expanding the result to order $\epsilon^{2}$ in the buffer region yields

$$
\begin{aligned}
& \mathrm{g}_{t t}=-f_{t t}+r^{2} H_{t t i_{1} i_{2}}^{(2,2)} \hat{n}^{i_{1} i_{2}}+r^{3} \sum_{\ell=2,3} H_{t t L}^{(3, \ell)} \hat{n}^{L} \\
& \mathrm{~g}_{t a}=r^{2} \sum_{\ell=1}^{3} H_{t a L}^{(2, \ell)} \hat{n}^{L}+r^{3} \sum_{\ell=1}^{4} H_{t a L}^{(3, \ell)} \hat{n}^{L} \\
& \mathrm{~g}_{a b}=f_{a b}+f \hat{n}_{a b}+r^{2} \sum_{\ell=0}^{4} H_{a b L}^{(2, \ell)} \hat{n}^{L}+r^{3} \sum_{\ell=0}^{5} H_{a b L}^{(3, \ell)} \hat{n}^{L} .
\end{aligned}
$$

Here $f_{t t}=1-\frac{2 \epsilon m}{r}+\frac{2 \epsilon^{2} m^{2}}{r^{2}}, f_{a b}=\left(1+\frac{2 \epsilon m}{r}+\frac{4 \epsilon^{2} m^{2}}{3 r^{2}}\right) \delta_{a b}$, and $f=\frac{\epsilon^{2} m^{2}}{r^{2}}$ describe the Schwarzschild metric in harmonic coordinates. The coefficients $H_{\mu \nu L}^{(2, \ell)}$ are functions of $\epsilon m / r$ forming linear combinations of $\mathcal{E}_{i j}(t)$ and $\mathcal{B}_{i j}(t)$, and $H_{\mu \nu L}^{(3, \ell)}$ are linear combinations of $\dot{\mathcal{E}}_{i j}(t), \dot{\mathcal{B}}_{i j}(t), \mathcal{E}_{i j k}(t)$, and $\mathcal{B}_{i j k}(t)$, where an overdot indicates a time-derivative. For example, $H_{t t i j}^{(2,2)}=-\left(1-\frac{5 \epsilon m}{3 r}+\frac{4 \epsilon^{2} m^{2}}{3 r^{2}}\right) \mathcal{E}_{i j}$.

If explicit appearances of $\mu$ are set to zero, the metric of Eqs. (13) - 15) reduces to that of a vaccum spacetime in Fermi coordinates centered on a geodesic, with $\mathcal{E}_{a b}=\mathcal{E}_{a b}^{(0)}+\epsilon \delta \mathcal{E}_{a b}+O\left(\epsilon^{2}\right), \mathcal{B}_{a b}=\mathcal{B}_{a b}^{(0)}+\epsilon \delta \mathcal{B}_{a b}+O\left(\epsilon^{2}\right)$, $\mathcal{E}_{a b c}=\mathcal{E}_{a b c}^{(0)}+O(\epsilon)$, and $\mathcal{B}_{a b c}=\mathcal{B}_{a b c}^{(0)}+O(\epsilon)$, where the zeroth-order fields $\mathcal{E}_{a b}^{(0)}, \mathcal{B}_{a b}^{(0)}$, etc., are components of $R_{\mu \nu \rho \sigma}$ and its first derivative evaluated at $r=0$. There is no manifest appearance of the fields $h_{\mu \nu}^{(n) \mathrm{R}}$ in Eqs. (13)-115); they are incorporated into the corrections $\delta \mathcal{E}_{a b}, \delta \mathcal{B}_{a b}$, etc. More significantly, there is no term corresponding to an acceleration; any such term would induce a mass-dipole-like term and would vanish in masscentered coordinates.

To match the expansions at orders $\epsilon$ and $\epsilon^{2}$, I seek a unique transformation $x^{\mu} \rightarrow x^{\mu}-\epsilon \xi^{(1) \mu}-\epsilon^{2} \xi^{(2) \mu}$ that brings the outer expansion into the form of Eqs. (13)(15). Decomposing $\xi^{(1) \mu}$ and $\xi^{(2) \mu}$ into irreducible STF pieces, one readily finds a unique transformation. At each order in $\epsilon$, this transformation can be thought of as putting the outer expansion into Fermi coordinates in $g_{\mu \nu}+\epsilon h_{\mu \nu}^{\mathrm{R}}$. The order- $\epsilon$ transformation is given in Ref. [7] up to order- $r$ terms in the metric; matching the metrics exhausts all freedom in that transformation and uniquely determines the standard result (10) for $a^{(1) \mu}$. Matching order- $\epsilon r^{2}$ terms in the metric fixes $\delta \mathcal{E}_{i j}$ and $\delta \mathcal{B}_{i j}$ in terms of $h_{\mu \nu}^{(1)}$. The order- $\epsilon^{2}$ transformation, when carried to order- $r$ terms in the metric, likewise uniquely determines

$$
\begin{aligned}
a_{i}^{(2)}= & \left.\frac{1}{2} h_{t t i}^{(2,1,1)}\right|_{a^{\mu}=0}-\left.h_{t i, t}^{(2,0,0)}\right|_{a^{\mu}=0}+h_{t t}^{(1,0,0)} a_{i}^{(1)} \\
& -\frac{1}{2} h_{t i}^{(1,0,0)} h_{t t, t}^{(1,0,0)}-\frac{11}{3} m \dot{a}_{i}^{(1)},
\end{aligned}
$$

where, as in Eq. (10), the evaluation at $a^{\mu}=0$ occurs only at time $t$. Summing $\epsilon a_{(1)}^{\mu}$ and $\epsilon^{2} a_{(2)}^{\mu}$, one finds, up 
to $O\left(\epsilon^{3}\right)$ errors,

$$
a^{\mu}=\frac{1}{2}\left(g^{\mu \nu}+u^{\mu} u^{\nu}\right)\left(g_{\nu}{ }^{\rho}-h_{\nu}^{\mathrm{R} \rho}\right)\left(h_{\sigma \lambda ; \rho}^{\mathrm{R}}-2 h_{\rho \sigma ; \lambda}^{\mathrm{R}}\right) u^{\sigma} u^{\lambda},
$$

where $h_{\mu \nu}^{\mathrm{R}}=\epsilon h_{\mu \nu}^{(1) \mathrm{R}}+\epsilon^{2} h_{\mu \nu}^{(2) \mathrm{R}}$. This is the geodesic equation in the locally defined regular metric $g_{\mu \nu}+h_{\mu \nu}^{\mathrm{R}}$, up to terms cubic in $h_{\mu \nu}^{\mathrm{R}} \cdot g_{\mu \nu}+h_{\mu \nu}^{\mathrm{R}}$ may trivially be extended to a $C^{n}$ (local) vacuum solution to the EFE, through order $\epsilon^{2}$, by finding the solution (7) through order $r^{n}$.

Equation (17) agrees with the form of Harte's equation of motion [22] but represents a major advance: it has been shown to apply to black holes, and it comes along with a concrete means of calculating both $a^{(2) \mu}$ and $h_{\mu \nu}^{(2)}$.

Discussion. I have shown that through second order in its mass, a small body moves on a geodesic of a certain locally defined regular metric. I have also derived results, given by (9), (12), and (16), that (together with the firstorder equations) may be used to simultaneously evolve the body's position and find the perturbation due to it, thereby solving the EFE through second order. Although these results were derived only for a nonrotating black hole, they should hold for any spherical, compact body with slow internal dynamics. For nonspherical bodies, they will be modified by higher multipole moments: $h_{\mu \nu}^{(2) \mathrm{R}}$ and $h_{\mu \nu}^{(2) \mathrm{S}}$ will be straightforwardly altered by the body's spin [20], and $a^{(2) \mu}$ will include well-known [22, 30, 31] couplings of the moments to the external curvature.

I wish to thank Leor Barack for many helpful discussions and suggested improvements to this manuscript. This work was supported by the Natural Sciences and Engineering Research Council of Canada.

[1] A. Einstein, L. Infeld, and B. Hoffmann, Annals of Mathematics 39, 65 (1938)

[2] Y. Mino, M. Sasaki, and T. Tanaka, Phys.Rev. D55, 3457 (1997)

[3] T. C. Quinn and R. M. Wald, Phys.Rev. D56, 3381 (1997)
[4] E. Poisson, A. Pound, and I. Vega, Living Rev. Relativity 14 (2011), http://www. livingreviews. org/lrr-2011-7

[5] S. E. Gralla and R. M. Wald, Class.Quant.Grav. 25, 205009 (2008)

[6] A. Pound, Phys. Rev. D 81, 024023 (2010)

[7] A. Pound, Phys. Rev. D 81, 124009 (2010)

[8] J. R. Gair, Class.Quant.Grav. 26, 094034 (2009)

[9] M. Favata, Phys.Rev. D83, 024028 (2011)

[10] A. Le Tiec, A. H. Mroue, L. Barack, A. Buonanno, H. P. Pfeiffer, et al., Phys.Rev.Lett. 107, 141101 (2011)

[11] A. Le Tiec, E. Barausse, and A. Buonanno(2011), arXiv:1111.5609

[12] T. Damour, Phys.Rev. D81, 024017 (2010)

[13] L. Barack, T. Damour, and N. Sago, Phys.Rev. D82, 084036 (2010)

[14] E. Barausse, A. Buonanno, and A. Le Tiec(2011), arXiv:1111.5610 [gr-qc]

[15] T. Hinderer and E. E. Flanagan, Phys.Rev. D78, 064028 (2008)

[16] D. A. Brown, J. Brink, H. Fang, J. R. Gair, C. Li, G. Lovelace, I. Mandel, and K. S. Thorne, Phys. Rev. Lett. 99, 201102 (2007)

[17] J. Abadie et al. (LIGO Scientific Collaboration, Virgo Collaboration), Class.Quant.Grav. 27, 173001 (2010)

[18] E. Rosenthal, Phys.Rev. D73, 044034 (2006)

[19] E. Rosenthal, Phys.Rev. D74, 084018 (2006)

[20] A. Pound(in preparation)

[21] S. Detweiler(2011), arXiv:1107.2098 [gr-qc]

[22] A. I. Harte(2011), arXiv:1103.0543 [gr-qc]

[23] J. Kevorkian and J. D. Cole, Multiple scale and singular perturbation methods (Springer, New York, 1996)

[24] W. Eckhaus, Asymptotic Analysis of Singular Perturbations (Elsevier North-Holland, New York, 1979)

[25] R. Kates, Ann. Phys. (N.Y.) 132, 1 (1981)

[26] T. Damour and L. Blanchet, Phil. Trans. R. Soc. Lond. A 320, 379 (1986)

[27] S. L. Detweiler and B. F. Whiting, Phys.Rev. D67, 024025 (2003)

[28] S. R. Dolan and L. Barack, Phys.Rev. D83, 024019 (2011)

[29] E. Poisson, Phys.Rev.Lett. 94, 161103 (2005)

[30] W. G. Dixon, Phil. Trans. Roy. Soc. Lond. A 277, 59 (1974)

[31] J. Steinhoff and D. Puetzfeld, Phys.Rev. D81, 044019 (2010) 